\title{
Safety, Tolerability and Pharmacokinetics of FAAH Inhibitor V158866: A Double-Blind, Randomised, Placebo-Controlled Phase I Study in Healthy Volunteers
}

\author{
Stephen Pawsey ${ }^{1,5} \cdot$ Mike Wood $^{1} \cdot$ Helen Browne $^{1} \cdot$ Kirsteen Donaldson $^{2} \cdot$ \\ Mark Christie $^{3} \cdot$ Steven Warrington ${ }^{4}$
}

Published online: 17 March 2016

(c) The Author(s) 2016. This article is published with open access at Springerlink.com

\begin{abstract}
Background and Objective The inhibition of fatty acid amide hydrolase 1 (FAAH) has been proposed as a novel mechanism for treating pain syndromes by increasing the levels of endogenous cannabinoids (ECs). This study describes the safety, tolerability, pharmacokinetics and pharmacodynamics of V158866, a reversible FAAH inhibitor, after first administration to man.

Methods 51 healthy male subjects were recruited into this double-blind, randomised, placebo-controlled, adaptive dose, phase I single (Part A) and repeated ascending dose (Part B) study. The primary outcome was the safety and tolerability of V158866. Secondary outcomes were (1) pharmacokinetics of V158866 and (2) pharmacodynamics of V158866, as assessed by changes in plasma EC concentrations.

Results Single oral doses of 5-300 mg and repeated oral doses of 50-500 mg were evaluated. V158866 was well tolerated, with no apparent treatment-related effects on laboratory variables. V158866 was rapidly absorbed with a mean terminal elimination half-life of 9.6-18.3 h (Day 7; Part B). V158866 reached steady state within 2-3 days of
\end{abstract}

Electronic supplementary material The online version of this article (doi:10.1007/s40268-016-0127-y) contains supplementary material, which is available to authorized users.

Mike Wood

m.wood@vernalis.com

1 Vernalis (R\&D) Ltd., 100 Berkshire Place, Wharfedale Road, Winnersh, Berkshire RG41 5RD, UK

2 Jade Consultants (Cambridge) Ltd., Cambridge, UK

3 Akranim Ltd., Maidstone, UK

4 Hammersmith Medicines Research, London, UK

5 Present Address: Circassia, Oxford, UK administration, with an accumulation ratio, based on $\mathrm{AUC}_{0-24 \mathrm{~h}}$, of approximately 2 on Day 7. V158866 showed a linear relationship between dose and AUC across the entire dose range. V158866 caused reversible, dose-related increases in plasma ECs. At hemi-equilibrium, there was a sigmoidal maximum effect relationship between plasma V158866 concentrations and changes in plasma ECs.

Conclusions V158866 is well tolerated, with linear pharmacokinetics suitable for once-daily administration, and reversible effects on plasma ECs. Maximum increases in plasma ECs occur with V158866 doses of 300-500 mg/day.

\section{Key Points}

Inhibition of fatty acid amide hydrolase 1 (FAAH) has been proposed as a novel mechanism for treating pain syndromes, by increasing the levels of endogenous cannabinoids. This approach has the potential to provide the pain relief of cannabis without the associated side effects.

This is a first in-man study of the FAAH inhibitor V158866. After oral administration, V158866 is well tolerated with predictable pharmacokinetics suitable for once-daily administration.

V158866 causes dose-dependent increases in plasma endocannabinoids, with maximum changes at doses of $300-500 \mathrm{mg} /$ day.

Further clinical trials in patients with syndromes such as neuropathic pain are needed to establish safety and efficacy. 


\section{Introduction}

The medicinal benefits and psychotropic disadvantages of cannabis (Cannabis sativa) have been recognized for centuries [1]. Cannabinoids, the pharmacologically active components of cannabis, act in vertebrates primarily on the G-protein-coupled post-synaptic receptors $\mathrm{CB}_{1}$ and $\mathrm{CB}_{2}$. $\mathrm{CB}_{1}$ receptors are predominantly located in the central nervous system (CNS), and additionally in tissues including the liver, adipose tissue, pancreas, gut, genital tract and muscle. Agonism typically causes cognitive impairment, hypolocomotion, hyperphagia, hypothermia, anticonvulsant activity and analgesia. Endogenous ligands at the $\mathrm{CB}_{1}$ and $\mathrm{CB}_{2}$ receptors include the endocannabinoids, anandamide ( $N$-arachidonyl ethanolamide, AEA) and 2-arachidonoyl-glycerol. Those receptors are also activated by $\Delta^{9}$ tetrahydrocannabinol (THC), the principal active substance in cannabis [2]. THC and other direct $\mathrm{CB}_{1}$ agonists (e.g. dronabinol, nabilone) have a range of clinical benefits, including analgesia (particularly for neuropathic pain [3]), anxiolysis, antidepressant and antiemetic properties, appetite stimulation and muscle relaxation [4, 5]. However, their clinical utility is limited by multiple side effects, such as impairment of cognition and motor control [6].

Fatty acid amide hydrolase (FAAH) is an integral membrane enzyme that hydrolyses the endocannabinoid, AEA, and related signalling lipids [7]. In humans, FAAH exists in two forms (FAAH1 and FAAH2 [8]). FAAHknockout (KO) mice display analgesia and anti-inflammatory phenotypes without disruption of motility, cognition or body temperature $[9,10]$, effects which are emulated by pharmacologic inhibition of FAAH [11, 12]. That has led to the hypothesis that FAAH inhibitors may be effective for the treatment of pain without the side effects associated with direct-acting cannabinoid agonists [13].

Several FAAH inhibitors are reported to be in development [14], but to our knowledge the only published clinical studies use the irreversible, urea-based inhibitor PF-04457845 [15]. PF-04457845 is well tolerated at doses that cause maximal inhibition of FAAH and elevation of plasma endocannabinoids [16] and was progressed into a phase II study in osteoarthritic pain where it was found to be without effect [17]. Whilst there has been some debate about the trial design [18], the lack of effect of PF04457845 in osteoarthritic pain argues for a more detailed review of the rationale for phase II clinical trials of FAAH inhibitors, with particular reference to the effects of cannabinoid agonists in patients with any particular pain state [19]. Indeed, as the beneficial clinical effects of cannabinoids are often due to their effects on comorbidities (rather than the perception of noxious stimuli per se), FAAH inhibition may be more appropriate for the treatment of pain syndromes such as neuropathic pain [3] and multiple sclerosis [5].

This is a report of the first clinical study of the azetidine analogue V158866, a potent, reversible, orally available FAAH inhibitor with efficacy in rodent models of inflammatory pain [20]. The primary objective of the study was to evaluate the safety and tolerability of single and repeated doses of V158866 in healthy male subjects. The secondary objectives of the study were to assess the pharmacokinetics and pharmacodynamics of V158866.

\section{Methods}

This was a single centre, phase I, double-blind, randomised, placebo-controlled, adaptive dose, single (Part A) and repeated ascending (Part B) dose study designed to compare the safety, tolerability, pharmacokinetics (PK) and pharmacodynamics (PD) of V158866 with placebo. The trial was conducted at Hammersmith Medicines Research (HMR), London, UK.

\subsection{Subjects}

Eligibility for this study required that subjects be healthy men aged 18-45 years, with a body mass index between 19 and $30 \mathrm{~kg} / \mathrm{m}^{2}$. Subjects had to be able to provide a semen sample and willing to use an effective method of contraception for the duration of the study and for 4 months after the last dose of study medication. All subjects had to be willing and able to comply with the requirements of the entire study, and provided written informed consent before participating.

Exclusion criteria included prior vasectomy, or the intention to father a child within 4 months of study conclusion, multiple drug allergies or allergy to any of the components of V158866 study medication and laboratory, electrocardiography (ECG) or other abnormalities that might increase the risk to the subject or interfere with the interpretation of study results. The remaining, standardized criteria related to cigarette smoking, alcohol consumption, recreational and therapeutic drug use (except paracetamol), and seropositivity for hepatitis B, hepatitis C or HIV. Subjects were not permitted to consume caffeine-containing food or drinks, grapefruit products, or alcohol for $24 \mathrm{~h}$ before or during each admission.

\subsection{Study Design}

The study was conducted between February and July 2011 in two parts, with Part B commencing after completion of Part A. 
Part A Single ascending dose, partial cross over: subjects in two cohorts of nine (six active and three placebo at each dose level) each received two single oral doses of V158866 and one dose of matching placebo in separate study periods, with a washout of at least 7 days between successive doses. Subjects participated in Part A for about 9 weeks.

Part B Repeated ascending dose, parallel group: subjects in four cohorts of eight (six active and two placebo at each dose level) each received once-daily doses of V158866 or placebo for 7 days. Subjects participated in Part B for about 6 weeks.

For each cohort, eligible subjects were assigned a sequential randomisation number on admission and treatment was allocated according to a pre-defined randomisation code generated by the Statistical Analysis System (SAS) programmer at HMR. Randomisation lists were not available to personnel at the study centre (with the exception of the Pharmacist and delegated study staff involved in dispensing), the Vernalis monitor, project statisticians or any of the project team at Vernalis until after database lock.

During Part A, between Part A and Part B, and during Part B of the study, the safety, PK and PD data from the previous dose cohort were reviewed in a blinded manner before recommendations for the next dose level were made.

\subsection{Dose Selection}

In non-clinical toxicology studies, the no observable adverse event level (NOAEL) dose of V158866 in rat and dog was associated with area under the concentration-time curve from time 0 to $24 \mathrm{~h}\left(\mathrm{AUC}_{0-24 \mathrm{~h}}\right)$ values of 13 and $87 \mu \mathrm{g} \cdot \mathrm{h} / \mathrm{mL}$, respectively. Using a safety factor of 100 and comparison with the NOAEL on a $\mathrm{mg} / \mathrm{kg}$ basis, or a safety factor of 10 and two different methods for predicting human exposure (PK modelling, and body surface area-corrected human equivalent dose), the estimated safe starting dose range based on the rat or dog was 2-7 mg and 25-150 mg, respectively. The starting dose of V158866 chosen in Part A of the study was $5 \mathrm{mg}$. As toxicological findings in the rat included minor reversible changes in the male reproductive tract, safety assessments included routine seminology. The doses selected for the remainder of the study were chosen on the basis of (blinded) emerging safety, PK and PD data to determine a safe and tolerable dose of V158866, maximum inhibition of FAAH and significant increases in plasma endocannabinoid concentrations.

\subsection{Safety}

Safety was assessed by monitoring adverse events (AEs) (coded using MedDRA version 14.1), recording vital signs (supine/semi-recumbent and standing blood pressure and pulse; respiratory rate and oral temperature), 12-lead ECGs, physical examination, body weight, laboratory safety tests (haematology, biochemistry, endocrine markers and urinalysis) and telemetry for cardiac rhythm monitoring. Semen quality was assessed at baseline and follow up.

\subsection{Bioanalytical Methods}

Venous blood samples were collected from each subject into lithium-heparinised tubes at intervals from pre-dose until 72 h (Part A) or 240 h (Part B) post-dose. Samples were centrifuged within 60 min of collection and the resultant plasma split into aliquots (for V158866 and endocannabinoid determination) and stored at $-20{ }^{\circ} \mathrm{C}$ or below until assay.

Urine was collected and pooled into 1-L polypropylene containers and stored at $4{ }^{\circ} \mathrm{C}$ for the duration of each specified collection interval (12 or $24 \mathrm{~h}$ ). Duplicate $4.5 \mathrm{~mL}$ aliquots of urine were then stored at $-20{ }^{\circ} \mathrm{C}$ until assay.

V158866 in human plasma and urine samples was assayed using a validated liquid chromatography with a tandem mass spectrometric (LC-MS/MS) method. Plasma samples were analysed for AEA, $N$-linoleoyl ethanolamide (LEA), $N$-oleoyl ethanolamide (OEA) and $N$-palmitoyl ethanolamide (PEA) using a validated LC-MS/MS method. Blood FAAH activity was determined by the method of Ahn et al. [21]. For all assays, samples in which the concentration of test agent exceeded the calibration range were diluted with blank assay matrix and re-analysed. The calibration ranges, Lower Limit of Quantitation (LLoQ) and accuracy values (based upon the calibration standards across the range) for each assay are detailed in the electronic supplementary material (Table ESM1).

\subsection{Test Product}

V158866 was presented as a liquid suspension $(2 \mathrm{mg} / \mathrm{mL}$ or $20 \mathrm{mg} / \mathrm{mL}$ ) in OraBlend ${ }^{\mathrm{TM}}$ suspending vehicle containing $5 \%$ weight/volume (w/v) polyvinylpyrrolidone (PVP, Kollidon K90) for oral administration, taken with $180 \mathrm{~mL}$ of water. Subjects were fasted for $10 \mathrm{~h}$ before each dose. Six dose levels $(5,15,30,70,150$ and $300 \mathrm{mg}$ ) of V158866 were administered in Part A as single doses. Four dose levels $(50,150,300$ and $500 \mathrm{mg}$ ) of V158866 were administered in Part B as daily doses for 7 days. Placebo was identical to the test product without the active ingredient.

\subsection{Data Analysis and Statistics}

No formal sample size calculation was performed as this was an exploratory study. Sample sizes of 6 V158866- 
treated subjects per dose level and 18 (Part A) or 8 (Part B) in the pooled placebo groups were considered sufficient to achieve the objectives of the study. No formal hypothesis testing was conducted.

Descriptive statistics [number of observations and mean and coefficient of variation $(\mathrm{CV})$ or median and range according to distribution] are presented for continuous PK and PD variables, with $95 \%$ confidence intervals (CI) where appropriate. Categorical data are summarised in frequency tables.

All available data were used in the PK and PD analyses, other than that from subjects with significant protocol deviations that might have invalidated or biased the results. To generate meaningful descriptive statistics for groups of analytical samples where the estimate of a test agent in some samples was below the LLoQ, a value equal to zero or LLoQ/2 was used.

The following non-compartmental PK parameters were calculated using WinNonlin version 6.2; maximum observed concentration $\left(C_{\max }\right)$, time to $C_{\max }\left(T_{\max }\right)$, terminal phase apparent elimination rate constant $\left(\lambda_{\mathrm{z}}\right)$, terminal elimination half-life $\left(t_{1 / 2}\right)$, area under the concentration-time curve from time 0 to $24 \mathrm{~h}\left(\mathrm{AUC}_{0-24 \mathrm{~h}}\right)$, from time zero to last measured value $\left(\mathrm{AUC}_{\text {last }}\right)$, from time zero extrapolated to infinity $\left(\mathrm{AUC}_{\mathrm{inf}}\right)$ and concentration observed $24 \mathrm{~h}$ post-dose on each day of Part B $\left(C_{\text {trough }}\right)$.

AUC and $C_{\max }$ were tested for dose proportionality by regression analysis of the log-transformed parameter versus log-transformed dose, with dose as a fixed effect and subject as a random effect. The slope, $\beta$, of this model was estimated with $90 \% \mathrm{CI}\left(\beta_{\mathrm{L}}-\beta_{\mathrm{U}}\right)$ and used to estimate the fold-increase in systemic exposure for a doubling in dose $(2 \beta)$ with $90 \% \mathrm{CI}\left(2 \beta_{\mathrm{L}}-2 \beta_{\mathrm{U}}\right)$. The CI of the slope, $\beta$, was compared with the limits 1.6 to 2.5 (i.e. double the standard equivalence limits of 0.8 to 1.25 ) for both $\mathrm{AUC}_{\mathrm{inf}}$ and $C_{\max }$.

Comparisons of the single dose PK parameters (Day 1) and those at the end of the repeated dose period (Day 7) were made using a mixed effects model on log transformed $\mathrm{AUC}_{0-24 \mathrm{~h}}$ and $C_{\max }$ including day and dose (classification factor) as fixed effects and subject as a random effect. The day-by-dose interaction term was also investigated. This model was used to estimate the accumulation ratio (Day 7/Day 1) with $95 \%$ CIs (from the back-transformed mean differences). The time to achieve steady state was explored graphically by plotting $C_{\text {trough }}$ values against study day. Concentration-response analysis was performed in Origin v8.1 using a non-linear curve fit maximum effect $\left(E_{\max }\right)$ model where $\beta$ was fixed at the baseline plasma concentration for each endogenous cannabinoid (EC), whilst $E_{\max }$ and midpoint locations were variable.

\section{Results}

\subsection{Baseline Demographics}

An overall summary of subject demographics is provided in the electronic supplementary material (Table ESM2). There were only minor differences between Parts of the study or between dose groups.

\subsection{Safety and Tolerability}

Adverse events In total, 19 subjects were treated in Part A. One subject discontinued after the first dose $(5 \mathrm{mg})$ owing to inadequate venous access. All remaining subjects each received two single doses of V158866 and one of placebo in separate treatment periods. All 32 subjects enrolled in Part B completed the study. The number of subjects experiencing at least one treatment-emergent adverse event (TEAE), the severity of TEAEs and their possible relationship to study medication are summarised in Table 1 .

V158866 was generally well tolerated with very few, mostly mild, TEAEs reported. There were no severe TEAEs, serious adverse events (SAEs) or TEAEs leading to treatment withdrawal. The frequency of TEAEs was generally similar across dose groups except for the highest repeated dose (500 $\mathrm{mg}$ daily) group, in which there were more overall, treatment-related and moderate TEAEs.

The most commonly reported treatment-related TEAEs were nervous system disorders (headache, dizziness and somnolence; Table 2). Events of that nature are consistent with CNS-active drugs but are also commonly reported after placebo in healthy volunteer studies. Overall, 13 were considered possibly related to study treatment.

TEAEs such as dizziness and orthostatic hypotension were reported mainly in the highest dose group $(500 \mathrm{mg}$ daily, Part B). Other TEAEs were generally consistent with those expected in a phase I trial or were single events showing no apparent trend.

Other findings related to safety: there were no apparent treatment- or dose-related effects of V158866 on routine laboratory safety tests, vital signs, ECG, telemetry or physical examination. Although seminology data were highly variable both within and between subjects, there was no apparent effect of V158866 on any seminology variable (sperm count, semen volume, motility, liquefaction or inflammatory cells).

\subsection{Pharmacokinetics}

Part A (single dose $P K$ ) The pharmacokinetic profile from 19 subjects [age range 19-42 years; mean weight \pm standard error of the mean $(\mathrm{SEM}) 78.7 \pm 2.8 \mathrm{~kg}$ ] is presented; 
Table 1 Summary of treatment-emergent adverse events (TEAEs)

\begin{tabular}{|c|c|c|c|c|c|c|c|}
\hline \multirow[t]{2}{*}{ Part A } & \multirow[t]{2}{*}{ Placebo } & \multicolumn{6}{|c|}{ Dose V158866 } \\
\hline & & $5 \mathrm{mg}$ & $15 \mathrm{mg}$ & $30 \mathrm{mg}$ & $70 \mathrm{mg}$ & $150 \mathrm{mg}$ & $300 \mathrm{mg}$ \\
\hline$n$ & 18 & 7 & 6 & 6 & 6 & 6 & 6 \\
\hline Subjects with $\geq 1$ TEAE & 7 & 6 & 2 & 3 & 2 & 2 & 2 \\
\hline Mild (moderate) TEAEs & $7(1)$ & $4(2)$ & $1(1)$ & $2(1)$ & 2 & 2 & $2(1)$ \\
\hline Treatment-related TEAEs & 1 & 0 & 0 & 1 & 1 & 1 & 2 \\
\hline \multirow[t]{2}{*}{ Part B } & \multicolumn{2}{|c|}{ Placebo } & \multicolumn{5}{|c|}{ Daily dose V158866 } \\
\hline & & & $50 \mathrm{mg}$ & $150 \mathrm{mg}$ & & $300 \mathrm{mg}$ & $500 \mathrm{mg}$ \\
\hline$n$ & 8 & & 6 & 6 & & 6 & 6 \\
\hline Subjects with $\geq 1$ TEAE & 3 & & 3 & 2 & & 3 & 6 \\
\hline Mild (moderate) TEAEs & & & $2(1)$ & 2 & & $2(1)$ & $5(3)$ \\
\hline Treatment-related TEAEs & 1 & & 3 & 1 & & 2 & 3 \\
\hline
\end{tabular}

Data are presented as number of subjects in each category

A TEAE was defined as an AE that started or increased in severity after the first dose of study medication up until the post-study visit Treatment-related TEAEs were those with a possible relationship to study medication

Table 2 System organ class of treatment-related treatment-emergent adverse events (TEAEs) reported by $\geq 2$ subjects in Part B of the study

\begin{tabular}{|c|c|c|c|c|c|c|}
\hline \multirow[t]{2}{*}{ System organ class } & \multirow[t]{2}{*}{ Preferred term } & \multirow[t]{2}{*}{ Placebo $(n=8)$} & \multicolumn{4}{|c|}{ Daily dose V158866 } \\
\hline & & & $50 \mathrm{mg}(n=6)$ & $150 \mathrm{mg}(n=6)$ & $300 \mathrm{mg}(n=6)$ & $500 \mathrm{mg}(n=6)$ \\
\hline \multirow[t]{3}{*}{ Nervous system } & Headache & & 2 & & 1 & 1 \\
\hline & Dizziness & & 1 & & & 1 \\
\hline & Somnolence & & & 1 & & 1 \\
\hline General & Fatigue & 1 & & & 1 & 1 \\
\hline
\end{tabular}

Data are presented as number of subjects in each category

one subject discontinued after the first dose $(5 \mathrm{mg})$ after difficulty with venous access, and was replaced. V158866 was rapidly absorbed (median $T_{\max } 1.5-2.5 \mathrm{~h}$ ) with doserelated increases in $C_{\max }$ and a multiphasic decline in plasma concentrations with mean terminal elimination half-lives in the range 5.4-11.2 h. A tendency for the plasma concentration of V158866 to increase between 18 and $24 \mathrm{~h}$ after administration was noted. Non-compartmental PK parameters are summarised in Table 3, and mean plasma concentrations are shown in Fig. 1a.

There was clear dose proportionality for $\mathrm{AUC}_{\mathrm{inf}}$ after single doses of V158866, with a 2.0-fold increase in $\mathrm{AUC}_{\text {inf }}(90 \%$ CI 1.9-2.1) for a doubling of V158866 (Fig. 1b) as tested using the power model (described in Sect. 2.7). The increase in $C_{\max }$ was somewhat less than dose proportional, with a 1.7 -fold increase $(90 \%$ CI 1.7-1.8) for each doubling of V158866 dose (electronic supplementary material; Fig. ESM3).

Little urinary excretion of V158866 was observed (proportion of dose excreted in urine $<0.3 \%$ ). As no human metabolites of V158866 were identified in vitro, and V158866 appeared to have relatively low plasma clearance in this study, plasma and urine samples were not analysed for metabolites.

Part $B$ (repeated dose $P K$ ) The data from all 24 V158866-treated subjects (age range 19-42 years; mean weight \pm SEM $76.6 \pm 1.5 \mathrm{~kg}$ ) are presented. The plasma profiles at Day 1 and Day 7 were generally similar to that in Part A, with concentrations peaking quickly and decreasing thereafter. The tendency for concentrations to increase at $18-24 \mathrm{~h}$ was more apparent at Day 7 than Day 1. Non-compartmental PK on Day 1 and Day 7 are summarised in Table 4, and mean plasma concentrations are shown in Fig. 2.

There is approximately twofold accumulation of V158866 after 7 days of administration at $150 \mathrm{mg}$ once daily and above. The calculated accumulation ratio between Days 1 and 7 for $150 \mathrm{mg} /$ day V158866 and above ranged from 1.5 (95\% CI 1.2-2.0) to $1.8(95 \% \mathrm{CI}$ 1.4-2.3) for $C_{\max }$ and from 1.9 (95\% CI 1.6-2.3) to 2.8 (95\% CI 2.3-3.4) for $\mathrm{AUC}_{0-24 \mathrm{~h}}$. Dose proportionality was demonstrated for $\mathrm{AUC}_{0-24 \mathrm{~h}}$ on Day 7 with a 2.0 -fold 
Table 3 Summary of V158866 pharmacokinetics following single oral doses (Part A)

\begin{tabular}{lllllll}
\hline Parameter & V158866 & & & & \\
\cline { 2 - 6 } & $5 \mathrm{mg}(n=6-7)$ & $15 \mathrm{mg}(n=6)$ & $30 \mathrm{mg}(n=6)$ & $70 \mathrm{mg}(n=6)$ & $150 \mathrm{mg}(n=6)$ & $300 \mathrm{mg}(n=6)$ \\
\hline$C_{\max }(\mathrm{ng} / \mathrm{mL})$ & $22.5(25.9)$ & $98.6(32.7)$ & $94.1(27.3)$ & $271.4(44.3)$ & $393.2(21.8)$ & $805.7(43.0)$ \\
& $18-32$ & $65-150$ & $68-123$ & $177-512$ & $306-504$ & $481-1440$ \\
$T_{\max }{ }^{\mathrm{a}}(\mathrm{h})$ & 1.50 & 1.75 & 1.75 & 2.00 & 1.52 & 2.50 \\
& $1.0-2.1$ & $0.5-3.0$ & $1.0-3.0$ & $1.0-3.0$ & $1.0-3.0$ & $1.5-3.0$ \\
$\mathrm{AUC}_{\text {inf }}(\mathrm{ng} . \mathrm{h} / \mathrm{mL})$ & $109.6(35.0)$ & $502.0(29.2)$ & $744.0(38.2)$ & $2125.0(47.4)$ & $3978.8(26.0)$ & $8680.1(18.4)$ \\
& $64-161$ & $326-699$ & $354-958$ & $1239-4181$ & $2676-5558$ & $7240-11471$ \\
$t_{1 / 2}(\mathrm{~h})$ & $5.40(37.6)$ & $10.01(79.7)$ & $10.05(77.1)$ & $9.24(19.8)$ & $11.20(65.1)$ & $9.07(30.7)$ \\
& $3.2-9.9$ & $3.9-19.7$ & $4.7-29.8$ & $7.4-12.0$ & $5.8-23.0$ & $6.0-13.5$ \\
$\lambda_{\mathrm{z}}(1 / \mathrm{h})$ & $0.13(37.6)$ & $0.07(79.7)$ & $0.07(77.1)$ & $0.08(19.8)$ & $0.06(65.1)$ & $0.08(30.7)$ \\
& $0.07-0.22$ & $0.04-0.18$ & $0.02-0.15$ & $0.06-0.09$ & $0.03-0.12$ & $0.05-0.12$
\end{tabular}

Data are presented as geometric mean (geometric $\mathrm{CV}$ ) and range unless specified otherwise

$\lambda_{z}$ terminal phase apparent elimination rate constant, $A U C_{i n f}$ area under the concentration-time curve from time extrapolated to infinity, $C_{m a x}$ maximum concentration, $t_{1 / 2}$ half-life, $T_{\max }$ time to maximum concentration

a $T_{\max }$ is presented as median and range

increase (90 \% CI 1.8-2.2) for a doubling dose as tested using the power model (described in Sect. 2.7). The increase in $C_{\max }$ on Day 7 was somewhat less than dose proportional, with a 1.7 -fold increase $(90 \%$ CI $1.5-1.8)$ for each doubling dose. Dose proportionality was not observed on Day 1 for either $C_{\max }$ or $\mathrm{AUC}_{0-24 \mathrm{~h}}$ as subsequent doses prevented estimation of the terminal half-life. Steady state $C_{\text {trough }}$ values appeared to be reached within 2-3 days postdose (Fig. 2).

Based on the low urinary excretion of V158866 observed in Part A, urine samples from Part B were not analysed.

\subsection{Pharmacodynamics}

Part A (single dose PD) V158866 appeared to cause maximal inhibition of blood FAAH activity at doses $\geq 30 \mathrm{mg}$. However, FAAH activity data were imprecise, so were unsuitable for more detailed PD analysis and are not reported here.

V158866 caused dose-related increases in plasma AEA, with measurable changes after the 5-mg dose (Fig. 3). EC changes followed the plasma V158866 concentration, with a time lag of about $2 \mathrm{~h}$ between maximum PK and PD responses, and a return to baseline at $72 \mathrm{~h}$ post-dose. V158866 caused similar changes in plasma OEA and PEA concentrations, but the LEA response was biphasic with a second peak at $12 \mathrm{~h}$ post-dose (electronic supplementary material; Fig. ESM4). Whilst the PD effects of V158866 were clearly reversible, there was a lag between maximum PK and PD responses such that when the relationship between mean plasma V158866 and AEA concentrations was plotted in time order, a characteristic counter- clockwise hysteresis loop was observed (electronic supplementary material; Fig. ESM5). Hysteresis was also seen for OEA and PEA, but not LEA, which was complicated by the biphasic PD response (data not shown).

Part B (repeated dose PD) As in Part A of the study, V158866 caused dose-related increases in plasma EC concentrations on both Day 1 and Day 7. EC changes followed the plasma V158866 concentration, with a time lag of about $2 \mathrm{~h}$ between maximum PK and PD responses, and a return to baseline after Day 7 by $72 \mathrm{~h}$ post-dose. During the repeated dose phase of Part B, steady state $\left(C_{\text {trough }}\right)$ plasma ECs were achieved within 2-3 days postdose and were sustained until Day 8. Plasma AEA changes are shown in Fig. 4; OEA and PEA showed qualitatively similar responses. The biphasic LEA response observed in Part A was again observed on Days 1 and 7 of Part B (electronic supplementary material; Fig. ESM6, ESM7 and ESM8).

A comparison of the $C_{\text {trough }}$ plasma concentrations of V158866 with the corresponding plasma EC concentration allowed a concentration-response curve to be generated for each $\mathrm{EC}$ at hemi-equilibrium. This relationship (which is best described by an $E_{\max }$ model) is shown in Fig. 5: V158866 caused concentration-dependent increases in plasma AEA, LEA, OEA and PEA concentrations, up to a maximum of approximately $3,4,8$ and $5 \mathrm{ng} / \mathrm{mL}$, respectively. Whilst the mid-point locations of the concentrationresponse curves vary somewhat (potency order; PEA $>$ OEA $>$ AEA $=$ LEA), this analysis indicated that maximum increases in AEA, OEA and PEA occur at plasma V158866 concentrations of around $300 \mathrm{ng} / \mathrm{mL}$ or greater, which can be achieved with oral V158866 doses of $300-500 \mathrm{mg} /$ day. 
Fig. 1 V158866 plasma exposure after single oral dose administration (Part A): mean (+SEM) concentration-time profiles (a), and relationship between dose and $\mathrm{AUC}_{\text {inf }}(\mathbf{b})$. $S E M$ standard error of the mean
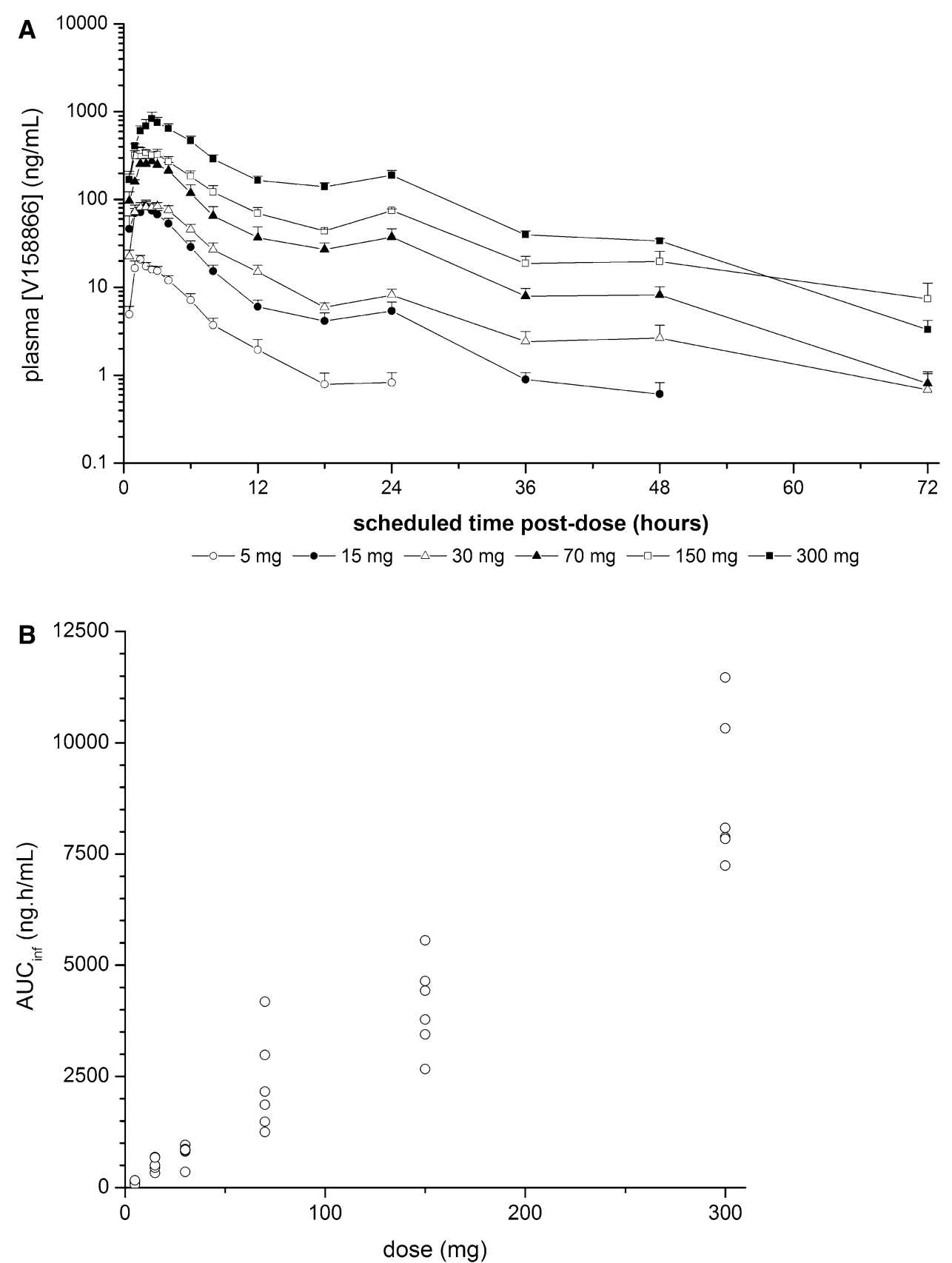

\section{Discussion}

This paper details the first in-human study of V158866, a potent, novel, reversible FAAH inhibitor. V158866 was well tolerated after single oral doses of 5-300 mg, and repeated oral doses of $50-500 \mathrm{mg}$ for up to 7 days. Treatment-emergent adverse events for V158866 after single and repeated doses were mostly mild in severity and similar to those reported for placebo, except perhaps at the highest repeated dose (500 mg daily). The most commonly reported adverse events were nervous system disorders, as might be expected for a CNS-active drug, and symptoms such as dizziness and orthostatic hypotension might have been treatment-related. This bland tolerability profile is consistent with both the phenotype of the FAAH KO mouse [9] and with previous clinical reports of the effects of FAAH inhibition [16]. V158866 had no effect on vital signs, ECG, routine laboratory safety tests or on any seminology variable.

After single oral doses, the pharmacokinetics of V158866 were predictable, with rapid absorption followed by elimination with a half-life in the range of 5.4-11.2 $\mathrm{h}$. 
Table 4 Summary of V158866 pharmacokinetics on Day 1 and Day 7 of administration (Part B)

\begin{tabular}{|c|c|c|c|c|c|c|c|c|}
\hline \multirow[t]{3}{*}{ Parameter } & \multicolumn{8}{|c|}{ V158866 (dose/day) } \\
\hline & \multicolumn{2}{|c|}{$50 \mathrm{mg}(n=6)$} & \multicolumn{2}{|c|}{$150 \mathrm{mg}(n=6)$} & \multicolumn{2}{|c|}{$300 \mathrm{mg}(n=6)$} & \multicolumn{2}{|c|}{$500 \mathrm{mg}(n=6)$} \\
\hline & Day 1 & Day 7 & Day 1 & Day 7 & Day 1 & Day 7 & Day 1 & Day 7 \\
\hline \multirow[t]{3}{*}{$C_{\max }(\mathrm{ng} / \mathrm{mL})$} & $273.9(32.2)$ & 311.9 (29.8) & $395.5(64.8)$ & $678.5(50.6)$ & $684.0(26.3)$ & 1040.3 (18.9) & \multirow{3}{*}{$\begin{array}{c}1009.6 \\
(24.9) \\
801-1400\end{array}$} & $1817.8(21.4)$ \\
\hline & $170-401$ & $215-447$ & $155-834$ & $304-1040$ & $460-1030$ & $862-1420$ & & $1310-2480$ \\
\hline & & & & & & & & \\
\hline \multirow[t]{2}{*}{$T_{\max }^{\mathrm{a}}(\mathrm{h})$} & 1.50 & 1.75 & 2.25 & 2.50 & 2.00 & 2.00 & 2.00 & 2.00 \\
\hline & $0.5-2.5$ & $1.0-4.0$ & $1.0-3.0$ & $1.0-4.0$ & $1.0-4.0$ & $1.5-4.0$ & $1.0-4.1$ & $1.5-2.5$ \\
\hline \multirow[t]{2}{*}{$C_{\text {trough }}{ }^{\mathrm{b}}(\mathrm{ng} / \mathrm{mL})$} & $23.9(20.5)$ & $25.7(48.1)$ & $59.2(65.1)$ & $122.9(72.7)$ & $144.6(28.7)$ & $276.5(32.2)$ & $174.6(28.4)$ & $479.4(38.7)$ \\
\hline & $17-30$ & $14-47$ & $19-90$ & $41-258$ & $106-218$ & $190-485$ & $122-239$ & $233-627$ \\
\hline \multirow[t]{2}{*}{$\begin{array}{l}\mathrm{AUC}_{0-24 \mathrm{~h}}(\mathrm{ng} \cdot \mathrm{h} / \\
\quad \mathrm{mL})\end{array}$} & $\begin{array}{r}1472.5 \\
(35.7)\end{array}$ & $\begin{array}{c}1956.4 \\
(30.3)\end{array}$ & $\begin{array}{r}2997.7 \\
(53.4)\end{array}$ & $\begin{array}{r}6051.6 \\
(47.7)\end{array}$ & $\begin{array}{c}5422.1 \\
(16.9)\end{array}$ & $\begin{array}{c}10248.2 \\
(26.7)\end{array}$ & $\begin{array}{c}6906.7 \\
(30.5)\end{array}$ & $\begin{array}{c}19286.1 \\
(29.2)\end{array}$ \\
\hline & 849-2090 & $1426-3099$ & $1803-6922$ & 4044-10920 & $4066-6572$ & $7666-14260$ & 4069-9827 & 11219-24845 \\
\hline \multirow[t]{2}{*}{$t_{1 / 2}(\mathrm{~h})$} & $6.08(20.6)$ & $9.58(13.1)$ & $9.35(44.1)$ & $10.42(28.8)$ & 9.77 (39.6) & $18.26(41.5)$ & $9.00(22.5)$ & $16.37(35.1)$ \\
\hline & $4.8-8.0$ & $7.6-11.0$ & $5.8-18.3$ & $8.0-15.5$ & $7.1-20.5$ & $11.3-33.4$ & $6.9-12.2$ & $11.9-31.0$ \\
\hline \multirow[t]{2}{*}{$\lambda_{\mathrm{z}}(1 / \mathrm{h})$} & $0.11(20.6)$ & $0.072(13.1)$ & $0.074(44.1)$ & $0.067(28.8)$ & $0.071(39.6)$ & $0.038(41.5)$ & $0.077(22.5)$ & $0.042(35.1)$ \\
\hline & $0.09-0.14$ & $0.06-0.09$ & $0.04-0.12$ & $0.05-0.09$ & $0.03-0.10$ & $0.02-0.06$ & $0.06-0.10$ & $0.02-0.06$ \\
\hline
\end{tabular}

Data are presented as geometric mean (geometric $\mathrm{CV}$ ) and range unless specified otherwise

$\lambda_{z}$ terminal phase apparent elimination rate constant, $A U C_{0-24 \mathrm{~h}}$ area under the concentration-time curve from time $0-24 \mathrm{~h}, C_{m a x}$ maximum concentration, $C_{\text {trough }}$ lowest concentration, $t_{1 / 2}$ half-life, $T_{\max }$ time to maximum concentration

a $T_{\max }$ is presented as median and range

${ }^{\mathrm{b}}$ Reported plasma $C_{\text {trough }}$ values were taken approximately $24 \mathrm{~h}$ after administration of V158866 on Day 1 and Day 7

Fig. 2 V158866 mean (+SEM) plasma concentration-time profiles after repeated oral administration (Part B). Plasma concentrations from Days 2-6 are pre-dose $\left(C_{\text {trough }}\right)$ estimates. $C_{\text {trough }}$ lowest concentration, SEM standard error of the mean

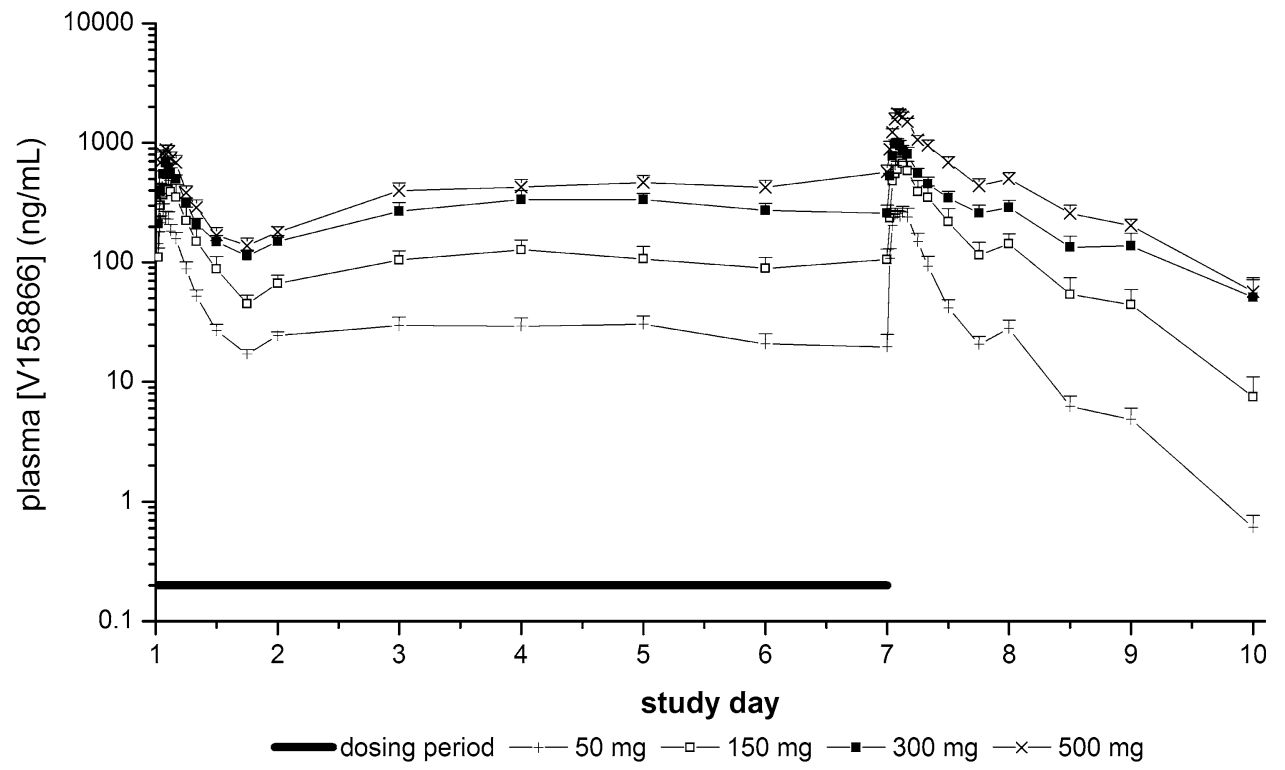

V158866 showed a linear relationship between dose and exposure across the entire dose range, with no evidence of the non-linear pharmacokinetics reported for PF-04457845 [16]. The repeated dose pharmacokinetics of V158866 are suitable for once-daily administration, with rapid absorption, a non-compartmental terminal elimination half-life in the range 9.6-18.3 $\mathrm{h}$ on Day 7, and clear dose-proportionality at the 7-day time point. V158866 reached steady state $C_{\text {trough }}$ values within 2-3 days of administration, with an accumulation ratio (at doses of $150 \mathrm{mg}$ and above, based on $\mathrm{AUC}_{0-24 \mathrm{~h}}$ ) of about twofold on Day 7. When halflife is estimated from the extent of accumulation in Part B, 
Fig. 3 AEA mean (+SEM) plasma concentrations after single oral dose administration (Part A). AEA $N$-arachidonyl ethanolamide, SEM standard error of the mean
Fig. 4 AEA mean (+SEM) plasma concentrations after repeated oral dose administration (Part B). Plasma concentrations from Days 2-6 are pre-dose $\left(C_{\text {trough }}\right)$ estimates. $A E A N$-arachidonyl ethanolamide, $C_{\text {trough }}$ lowest concentration, SEM standard error of the mean
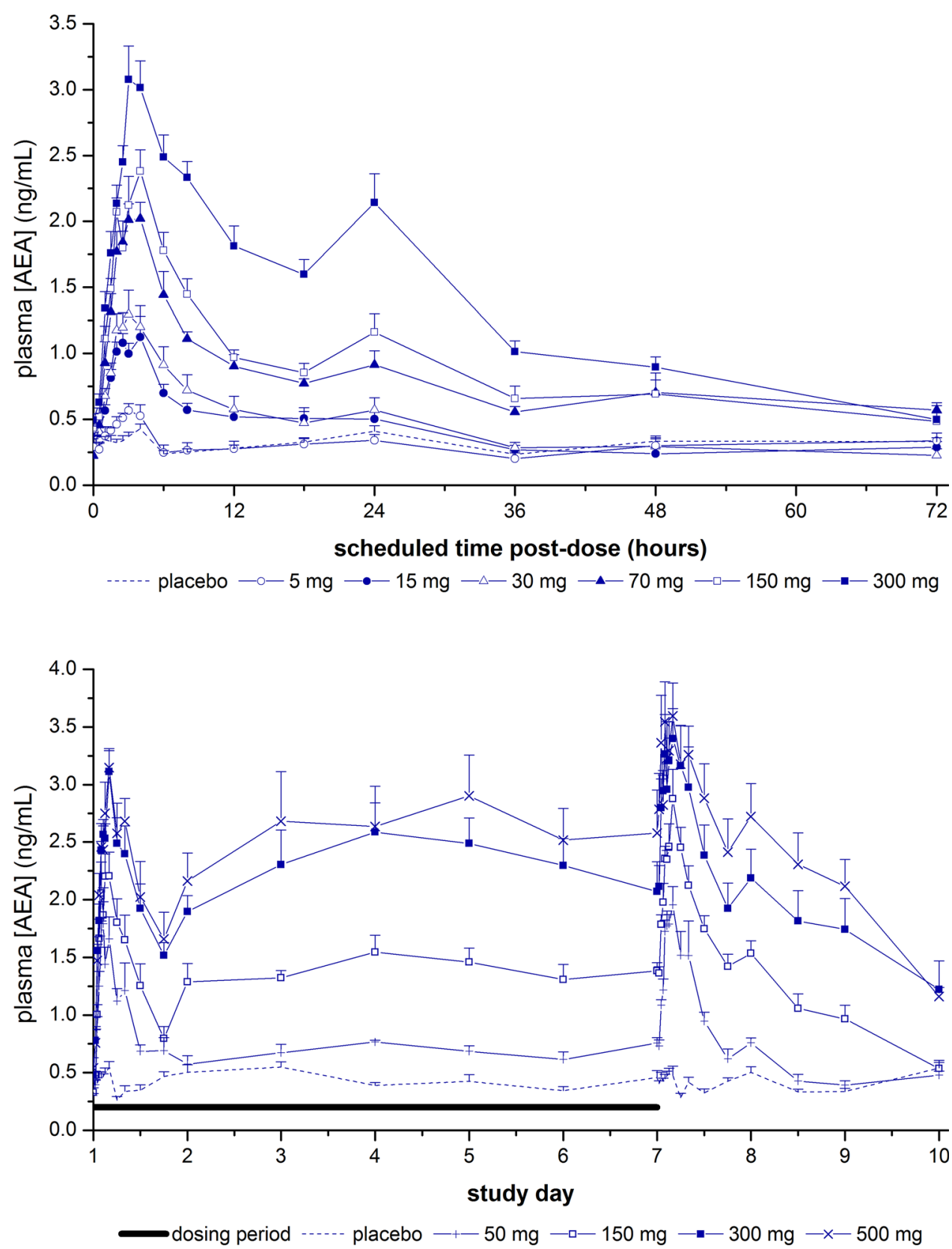

the approximately twofold accumulation of V158866 is consistent with the terminal elimination half-lives determined in Part B.

There was a tendency for the plasma concentration of V158866 to increase between 18 and $24 \mathrm{~h}$ after administration in both Part A and Part B. The reason for that is unclear. Whilst it is possible that V158866 is subject to enterohepatic recirculation, we would expect such a peak in plasma concentrations to occur earlier in the PK profile or after breakfast at $24 \mathrm{~h}$. However, as faecal samples were not collected, we have no evidence for the presence of enterohepatic recirculation. As V158866 is more soluble at high $\mathrm{pH}$, it is also possible that further absorption of
V158866 from the large intestine occurred between 18 and $24 \mathrm{~h}$ after oral administration.

We had initially intended to measure the pharmacodynamics of V158866 using two mechanistic biomarkers: inhibition of FAAH, and changes in EC concentrations as indicators of FAAH inhibition. However, in our hands, the blood FAAH inhibition assay was not precise enough: whilst it provided a clear 'all-or-none' marker of FAAH inhibition (which was complete at doses of $30 \mathrm{mg}$ and above in Part A and $50 \mathrm{mg}$ in Part B), it was unsuitable for more detailed PD analysis. As V158866 acts as an inhibitor of FAAH in blood with an $\mathrm{IC}_{50}$ (half maximal inhibitory concentration) of $\sim 60 \mathrm{nM}$ (data not shown), we relied on 
Fig. 5 Relationship between mean $C_{\text {trough }}$ plasma V158866 and endocannabinoid concentrations after repeated oral doses (Part B): the sigmoidal $E_{\max }$ line of best fit is shown for each endogenous cannabinoid. Combined data from all dose groups are shown; the 24-h post-dose time point was considered to be the $C_{\text {trough }}$ value for each study day. $A E A$ $\mathrm{N}$-arachidonyl ethanolamide, $C_{\text {trough }}$ lowest concentration, $E_{\text {max }}$ maximal effect, $L E A N$ linoleoyl ethanolamide, $O E A N$ oleoyl ethanolamide, PEA $N$ palmitoyl ethanolamide

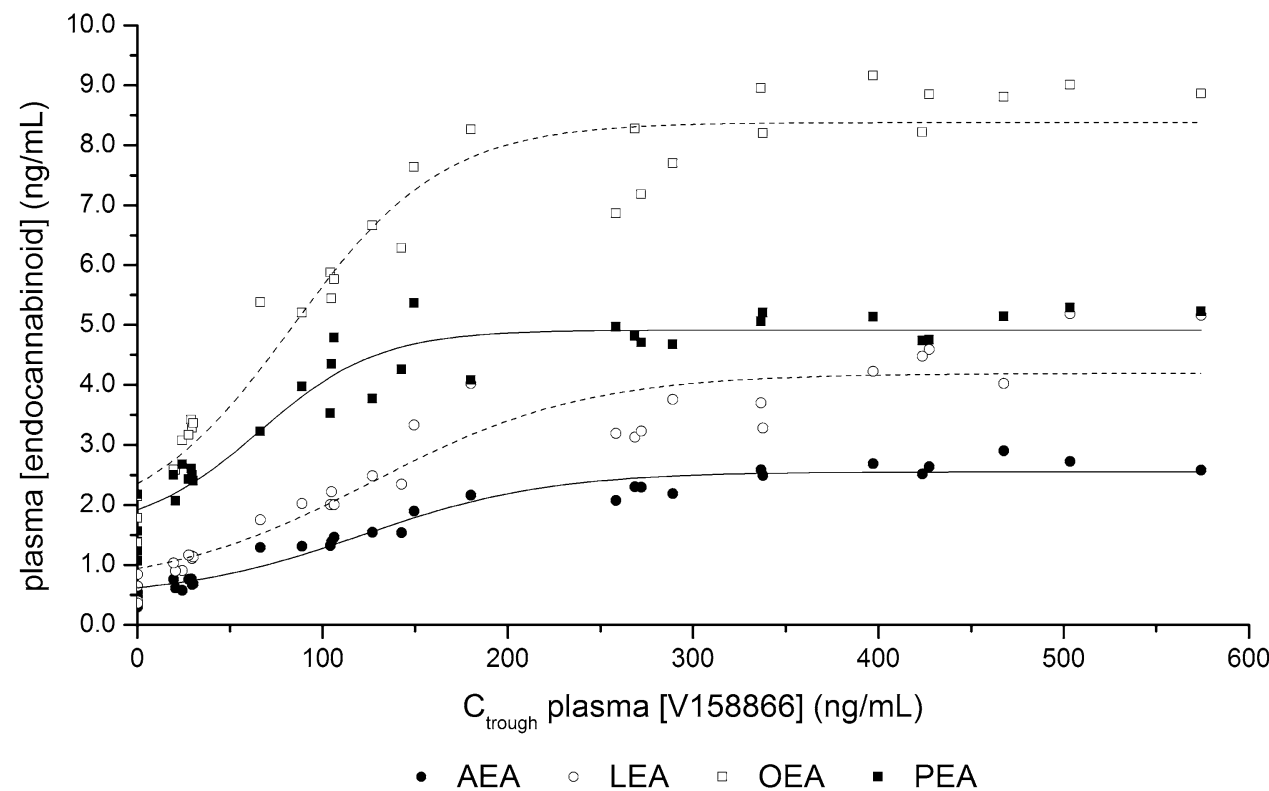

We chose to analyse the dose-response relationship of V158866 at the $C_{\text {trough }}$ values in Part B, as this approach allowed a concentration-response curve to be generated for each $\mathrm{EC}$ at hemi-equilibrium and minimized the influence of hysteresis [23]. V158866 caused dose-related increases in plasma AEA, LEA, OEA and PEA concentrations, with the plasma concentration effect best described by a sigmoidal $E_{\max }$ relationship. The potency of V158866 differs according to EC (PEA $>$ OEA $>$ AEA $=$ LEA), which appears to reflect the differential substrate specificity of FAAH [24], and argues for the use of more than one EC as a plasma biomarker in future studies. Nevertheless, we are confident that V158866 is capable of inducing maximal increases in plasma ECs for three reasons: firstly, the relationship between plasma V158866 and EC concentrations appears to reach an $E_{\max }$ (Fig. 5); secondly, at Day 7 of administration (Part B), further increases in plasma V158866 from $C_{\text {trough }}$ to $C_{\max }$ at 300 and $500 \mathrm{mg} / \mathrm{day}$ (Fig. 2) cause only moderate increases in plasma AEA (Fig. 4), and minimal changes in OEA and PEA concentrations, whilst LEA concentrations are doubled (electronic supplementary material, Fig. ESM6); and thirdly, the maximum changes in plasma AEA, LEA, OEA and PEA concentrations (approximately $3,4,8$ and $5 \mathrm{ng} / \mathrm{mL}$, respectively), are very similar to the maximum values reported for PF-04457845 in a similar study [16].

\section{Conclusions}

This report summarises the first in-man study of the novel, reversible FAAH inhibitor, V158866. V158866 had a good tolerability profile, linear pharmacokinetics suitable for 
once-daily administration, and reversible effects on plasma endocannabinoids. Our analysis indicates that maximum increases in AEA, OEA and PEA occurred at plasma V158866 concentrations of around $300 \mathrm{ng} / \mathrm{mL}$ or greater, which were achieved with oral V158866 doses of 300-500 mg/day.

Author Contributions Concept and design of study: SP, MW, KD, MC, SW. Conduct of study and preparation of clinical study report: SP, KD, SW. Data analysis and interpretation: SP, MW, HB, MC, SW. Writing of the manuscript: MC, HB. All authors discussed the results and commented on the manuscript.

\section{Compliance with Ethical Standards}

Funding This study was funded by Vernalis (R\&D) Ltd. Study design, data analysis, operational conduct, and manuscript preparation were overseen by Vernalis.

Conflict of interest SP, MW and HB are current/past employees of, and have shares/share options in Vernalis (R\&D) Ltd. KD is an employee of Jade Consultants (Cambridge) Ltd., whilst MC is an employee of Akranim Ltd., both of which are contracted to Vernalis. SW is an employee of Hammersmith Medicines Research, which was contracted to run the study on behalf of Vernalis. Intellectual property rights associated with V158866 and this clinical study are owned by Vernalis (R\&D) Ltd.

Ethical approval The study was conducted in accordance with the guidelines of the International Conference on Harmonization-Good Clinical Practice, and the 1964 Helsinki declaration. The protocol was approved by the Medicine and Healthcare Products Regulatory Agency (MHRA) and by the Edinburgh Independent Ethics Committee for Medical Research (REC reference number: 11/IE/0004). The trial was registered on ClinicalTrials.gov; ID NCT0163452.

Informed consent Written informed consent was obtained from all individual participants included in this study.

Open Access This article is distributed under the terms of the Creative Commons Attribution-NonCommercial 4.0 International License (http://creativecommons.org/licenses/by-nc/4.0/), which permits any noncommercial use, distribution, and reproduction in any medium, provided you give appropriate credit to the original author(s) and the source, provide a link to the Creative Commons license, and indicate if changes were made.

\section{References}

1. Pertwee RG. Cannabinoid pharmacology: the first 66 years. Br J Pharmacol. 2006;147:S163-71.

2. Mechoulam R. Discovery of endocannabinoids and some random thoughts on their possible roles in neuroprotection and aggression. Prostaglandins Leukot Essent Fatty Acids. 2002;66:93-9.

3. Dray A. Neuropathic pain: emerging treatments. Br J Anaesth. 2008;101:48-58.

4. Pertwee RG. Cannabis and cannabinoids: pharmacology and rationale for clinical use. Forsch Komplementarmed. 1999;6:12-5.
5. Smith PF. Cannabinoids in the treatment of pain and spasticity in multiple sclerosis. Curr Opin Investig Drugs. 2002;3:859-64.

6. Campbell FA, Tramèr MR, Carroll $\mathrm{D}$, et al. Are cannabinoids an effective and safe treatment option in the management of pain? A qualitative systematic review. BMJ. 2001;323:13-6.

7. Giang DK, Cravatt BF. Molecular characterization of human and mouse fatty acid amide hydrolases. Proc Natl Acad Sci USA. 1997;94:2238-42.

8. Wei BQ, Mikkelsen TS, McKinney MK, et al. A second fatty acid amide hydrolase with variable distribution among placental mammals. J Biol Chem. 2006;281:36569-78.

9. Lichtman AH, Shelton CC, Advani T, Cravatt BF. Mice lacking fatty acid amide hydrolase exhibit a cannabinoid receptor mediated phenotypic hypoalgesia. Pain. 2004;109:319-27.

10. Massa F, Marsicano G, Hermann H, et al. The endogenous cannabinoid system protects against colonic inflammation. J Clin Invest. 2004;113:1202-9.

11. Holt S, Comelli F, Costa B, Fowler CJ. Inhibitors of fatty acid amide hydrolase reduce carrageenan-induced hind paw inflammation in pentobarbital-treated mice: comparison with indomethacin and possible involvement of cannabinoid receptors. Br J Pharmacol. 2005;146:467-76.

12. Chang L, Luo L, Palmer JA, et al. Inhibition of fatty acid amide hydrolase produces analgesia by multiple mechanisms. Br J Pharmacol. 2006;148:102-13.

13. Cravatt BF, Lichtman AH. Fatty acid amide hydrolase: an emerging therapeutic target in the endocannabinoid system. Curr Opin Chem Biol. 2003;7:469-75.

14. Bisogno T, Maccarrone M. Latest advances in the discovery of fatty acid amide hydrolase inhibitors. Expert Opin Drug Discov. 2013;8:509-22.

15. Johnson DS, Stiff C, Lazerwith SE, et al. Discovery of PF04457845: A Highly Potent, Orally Bioavailable, and Selective Urea FAAH Inhibitor. ACS Med Chem Lett. 2011;2:91-6.

16. Li GL, Winter H, Arends R, et al. Assessment of the pharmacology and tolerability of PF-04457845, an irreversible inhibitor of fatty acid amide hydrolase-1, in healthy subjects. Br J Clin Pharmacol. 2012;73:706-16.

17. Huggins JP, Smart TS, Langman S, et al. An efficient randomized, placebo-controlled clinical trial with the irreversible fatty acid amide hydrolase-1 inhibitor PF-04457845, which modulates endocannabinoids but fails to induce effective analgesia in patients with pain due to osteoarthritis of the knee. Pain. 2012;153:1837-46.

18. Di Marzo V. Inhibitors of endocannabinoid breakdown for pain: not so FA(AH)cile, after all. Pain. 2012;153:1785-6.

19. Lichtman AH, Chapman V. A FAAH-fetched approach to treat osteoarthritis pain. Pain. 2011;152:959-60.

20. Roughley, S, Walls, S, Hart, T, et al. Azetidine derivatives. International patent WO2009/109743.

21. Ahn K, Johnson DS, Mileni M, et al. Discovery and characterization of a highly selective FAAH inhibitor that reduces inflammatory pain. Chem Biol. 2009;16:411-20.

22. Hansen HS, Diep TA. $N$-acylethanolamines, anandamide and food intake. Biochem Pharmacol. 2009;78:553-60.

23. Campbell DB. The use of kinetic-dynamic interactions in the evaluation of drugs. Psychopharmacology (Berl). 1990;100:433-50.

24. Ueda N, Puffenbarger RA, Yamamoto S, Deutsch DG. The fatty acid amide hydrolase (FAAH). Chem Phys Lipids. 2000;108:107-21. 